\title{
Early metastatic colorectal cancers show increased tissue expression of miR-17/92 cluster members in the invasive tumor front
}

Jepsen, Rikke Karlin; Novotny, Guy Wayne; Klarskov, Louise Laurberg; Bang-Bertelsen, Claus Heiner; Haakansson, Ida Trondhjem; Hansen, Anker; Christensen, Ib Jarle; Ass, Lene Buhl Riis; Høgdall, Estrid

Published in:

Human Pathology

Link to article, DOI:

10.1016/j.humpath.2018.05.027

Publication date:

2018

Document Version

Peer reviewed version

Link back to DTU Orbit

Citation (APA):

Jepsen, R. K., Novotny, G. W., Klarskov, L. L., Bang-Bertelsen, C. H., Haakansson, I. T., Hansen, A., Christensen, I. J., Ass, L. B. R., \& Høgdall, E. (2018). Early metastatic colorectal cancers show increased tissue expression of miR-17/92 cluster members in the invasive tumor front. Human Pathology, 80, 231-238. https://doi.org/10.1016/j.humpath.2018.05.027

\section{General rights}

Copyright and moral rights for the publications made accessible in the public portal are retained by the authors and/or other copyright owners and it is a condition of accessing publications that users recognise and abide by the legal requirements associated with these rights.

- Users may download and print one copy of any publication from the public portal for the purpose of private study or research.

- You may not further distribute the material or use it for any profit-making activity or commercial gain

- You may freely distribute the URL identifying the publication in the public portal 


\section{Accepted Manuscript}

Early metastatic colorectal cancers show increased tissue expression of miR-17/92 cluster members in the invasive tumor front

Rikke Karlin Jepsen, Guy Wayne Novotny, Louise Laurberg Klarskov, Claus Heiner Bang-Bertelsen, Ida Trondhjem Haakansson, Anker Hansen, Ib Jarle Christensen, Lene Buhl Riis

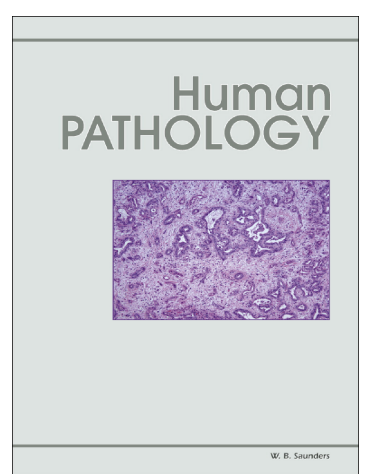
Ass, Estrid Høgdall

PII: S0046-8177(18)30207-7

DOI: doi:10.1016/j.humpath.2018.05.027

Reference: YHUPA 4593

To appear in: Human Pathology

Received date: 10 February 2018

Revised date: 28 April 2018

Accepted date: 22 May 2018

Please cite this article as: Rikke Karlin Jepsen, Guy Wayne Novotny, Louise Laurberg Klarskov, Claus Heiner Bang-Bertelsen, Ida Trondhjem Haakansson, Anker Hansen, Ib Jarle Christensen, Lene Buhl Riis Ass, Estrid Høgdall, Early metastatic colorectal cancers show increased tissue expression of miR-17/92 cluster members in the invasive tumor front. Yhupa (2018), doi:10.1016/j.humpath.2018.05.027

This is a PDF file of an unedited manuscript that has been accepted for publication. As a service to our customers we are providing this early version of the manuscript. The manuscript will undergo copyediting, typesetting, and review of the resulting proof before it is published in its final form. Please note that during the production process errors may be discovered which could affect the content, and all legal disclaimers that apply to the journal pertain. 


\section{Title}

\section{Early metastatic colorectal cancers show increased tissue expression of miR- $17 / 92$ cluster members in the invasive tumor front}

\section{Short running title}

Increased miR-17/92 in metastatic colorectal cancer

\section{Keywords}

Colorectal cancer, pT1, invasive tumor front, metastases, microarray, microRNA, miR-17/92,

\section{Author names and affiliations}

MD, Rikke Karlin Jepsen ${ }^{\text {a1 }}$

MSc PhD, Guy Wayne Novotny ${ }^{\mathrm{a}}$

$\mathrm{MD}, \mathrm{PhD}$, Louise Laurberg Klarskov $^{\mathrm{a}}$

MSc, PhD, Claus Heiner Bang-Bertelsen ${ }^{c}$

Ida Trondhjem Haakansson $^{\text {b }}$

Anker Hansen $^{\text {b }}$

MSc, Ib Jarle Christensen ${ }^{\text {a }}$

Ass. Professor, MD, PhD, Lene Buhl Riis $^{\text {a }}$

Professor, DMSc, Estrid $\underline{\mathrm{H} \emptyset \mathrm{gdall}}{ }^{\mathrm{a}}$ rikke.karlin.jepsen.01@regionh.dk

guy.wayne.novotny@regionh.dk

louise.laurberg.klarskov@regionh.dk

claban@food.dtu.dk

ith@medical-prognosis.com

ah@medical-prognosis.com

lb.Jarle.Christensen@regionh.dk

Lene.Buhl.Riis@regionh.dk

Estrid.Hoegdall@regionh.dk

${ }^{a}$ Department of Pathology and Molecular Unit, University of Copenhagen, Herlev and Gentofte Hospital, Herlev, Denmark

${ }^{\mathrm{b}}$ Medical Prognosis Institute A/S, Hørsholm, Denmark

${ }^{\mathrm{c} T e c h n i c a l ~ U n i v e r s i t y ~ o f ~ D e n m a r k, ~ N a t i o n a l ~ f o o d ~ i n s t i t u t e, ~ R e s e a r c h ~ G r o u p ~ f o r ~ M i c r o b i a l ~ B i o t e c h n o l o g y ~ a n d ~}$ Biorefining, Kgs Lyngby, Denmark

${ }^{1}$ Address from March $1^{\text {st }}$ 2018: Dept. Pathology, University of Copenhagen, Rigshospitalet, Copenhagen, Denmark. Email: rikke.karlin.jepsen@regionh.dk. Phone: +45 22316656

\section{Corresponding author}

Rikke Karlin Jepsen is the corresponding author and will handle correspondence at all stages of refereeing and publication. Phone: +45 22316656, Email from March $1^{\text {st }}$ 2018: rikke.karlin.jepsen@regionh.dk.

\section{Conflicts of interest}

$\mathrm{AH}$ and ITH are employees at Medical Prognosis Institute Aps (MPI). MPI did not offer any financial support or incentives and was not engaged in the scientific content of this paper other than performing the microarray analyses and assisting in the processing of raw expression data. AH and ITH did not have any influence on statistical analyses, interpretation of results or the decision to submit the manuscript for publication. All other authors declare no conflicts of interest.

\section{Funding}

This work was funded by Beckett Foundation [38069/38079], The A.P. Møller Foundation for the Advancement of Medical Science [14-187], Krista and Viggo Petersens Foundation [5793/44], Fabrikant Einer Willumsen Foundation [6000073] and Vald Foersom and Thyra Foersoms Foundation [41452-001]. Funding sources had no influence in any part of the study. 


\section{AbStract}

Accurate prediction of regional lymph node metastases (LNM) in endoscopically resected pT1 colorectal cancer (CRC) is crucial in treatment stratification for subsequent radical surgery. Several miRNAs have been linked to CRC invasion and metastasis, including the oncogenic miR-17/92 cluster, and expression levels might have predictive value in the risk assessment of early metastatic progression in CRC. We performed global miRNA microarray using tissue samples from the invasive front of pT1 CRC and investigated associations of the miR-17/92 cluster and presence of LNM.

In total, 56 matched pT1 CRCs were thoroughly clinico-pathologically characterized and miRNA microarrays were performed on invasive front tissue samples. Global miRNA intensities were screened using paired ttests between $\mathrm{pT} 1 \mathrm{pN}+$ and $\mathrm{pT} 1 \mathrm{pN} 0$. Associations between miR-17/92 and histopathological features were analyzed using general linear models and tumor cell adjusted expression intensities.

miR-17-3p and miR-92a were significantly higher expressed in the invasive front of tumors with LNM compared to those without, corresponding to 1.53 fold higher expression of miR-17-3p (95\%Cl: 1.04-2.24, $\mathrm{p}=0.030)$ and 1.28 fold higher expression of miR-92a $(95 \% \mathrm{Cl}: 1.01-1.68, p=0.042)$. An inverse association between miR-19a and presence of high grade tumor budding was observed ( 1.55 fold, $95 \% \mathrm{Cl}$ : 1.13-2.12, $p=0.008$ ). We provide evidence for associations between early regional LNM and high expression levels of the miR-17/92 cluster members: miR-17-3p and miR-92a, in the invasive front of CRC. Our results support a role for the miR-17/92 cluster in early metastatic progression of CRC and calls for further investigation. 


\section{INTRODUCTION}

Colorectal cancer (CRC) is the third most frequent cancer with 1.3 million annual new cases worldwide, and is a major cause of death and morbidity [1]. Risk of death is highly linked to the presence of lymph node metastases (LNM): A five year relative survival rate of 90.3\% in American patients with CRC detected in the early localized stage is reduced to $70.3 \%$ in patients with LNM and further reduced to $12.5 \%$ in patients with distant metastases [2]. Screening initiatives have increased the proportion of early stage CRCs, resulting in an increased proportion of endoscopically removed early cancers, pT1 CRCs. [3]. For those, reliable and accurate assessment of metastatic risk is crucial for decisions on subsequent radical surgery in order to remove possible local LNM and avoid dissemination of the disease. Patients with regional LNM have stage III disease and will benefit from subsequent radical surgery and adjuvant chemotherapy, while patients with no LNM have stage I disease and are with no indication for further treatment [4].

Guidelines for assessment of high risk in early CRC is based on histopathological features evaluated by microscopic evaluation of tumor slides, such as differentiation, lympho-vascular or venous invasion and depth of invasion [4] and recently the International Tumor Budding Consensus Conference (ITBCC 2016) recommendations suggested tumor budding as an additional prognostic and/or predictive factor to support treatment stratification of patients with endoscopically removed pT1 CRC [5]. In CRC budding tumor cells are thought to represent the morphological phenotype of cells undergoing epithelial-mesenchymal transition (EMT) [6], the biological process where epithelial polarized cells convert to assume a mesenchymal phenotype with motile and invasive properties, a crucial step in the malignant process of invasion and metastasis [7]

Despite histopathological risk assessment only $9-17 \%$ of patients with resected pT1 CRC have regional LNM at the time of diagnosis $[8,9]$. Since radical colorectal surgery carries a significant morbidity rate and postoperative mortality rate (of 3-6\%) [10] it is of major importance to identify patients who will actually benefit from surgery.

MicroRNAs (miRNAs) are a class of small non-coding RNA fragments (18-24 nucleotides in length) epigenetically regulating numerous cellular pathways including pathways involved in cancer initiation, development and progression. In CRC, expression of several specific miRNAs have been correlated to tumor cell invasion and metastasis [11,12], prognosis and therapeutic outcome [13] and recently Kwon Jung et al suggested a three-miRNA classifier for predicting LNM based on miRNA expression analyses in three small independent cohorts of pT1 CRC [14]. Additionally profiling studies have uncovered a number of miRNAs implicated in EMT $[11,12]$.

Members of the miR-17/92 cluster have been linked to EMT-like phenotypic cellular changes in colorectal cancer mainly through actions on PTEN $[15,16]$, and TNF $\alpha$ [17] as well as pro-metastatic remodeling of the extracellular matrix [18]. Additionally, the cluster is described involved in the early tumor development from normal mucosa to adenoma [19] and plays a role in tumor angiogenesis [18, 20], growth and inhibition of apoptosis [20, 21].

The miR-17/92 cluster is located on chromosome $13 q 31.3$ and transcriptional activation generates a single polycistronic primary transcript yielding six mature miRNAs: miR-17 (miR-17-3p and miR-17-5p), miR-18a, miR-19a, miR-20a, miR-19b, and miR-92a, involved in both normal- and cancer development through broad effects on various targets [22]. miR-92a has been described as the key oncogenic component of the cluster in CRC [21] and overexpression of miR-92a has been correlated to various clinico-pathological features such 
as advanced TNM stage, tumor size, lymphatic invasion, venous invasion, LNM and distant metastases [15, $16,23,24]$. In a previous study, despite demonstration of substantial intratumor expression heterogeneity, we were able to show differential expression of miR-92a in the invasive front of pT1/pT2 CRC compared to the main tumor body, indicating possible involvement of miR-92a in early CRC progression. However, we were not able to demonstrate significant associations to the presence of regional LNM in that cohort [25].

To further investigate the miR-17/92 cluster in metastatic progression in early PT1 CRC and to identify other potential miRNAs involved in the metastatic process of CRC, we performed global miRNA microarray profiling on invasive front tumor samples from a thoroughly clinico-pathologically characterized cohort of 28 pT1 CRCs with regional LNM matched with 28 LNM negative pT1 CRCs.

\section{MATERIAL AND METHODS}

\subsection{Flow of inclusion and exclusion}

Forty-four patients with resected primary metastasizing pT1 CRC, diagnosed at Herlev- and Gentofte University Hospital in the period 1.1.2009-31.01.2016 were identified in the local pathology registry. Twenty-eight of the 44 patients were included in the study cohort after microscopic reassessment and review of pathology reports. Fifteen patients were excluded due to the following: Age less than 50 years at diagnosis, more advanced tumor stage identified during reassessment, synchronous/metachronous CRCs or other infiltrating cancers and no/insufficient available tumor tissue for molecular analyses. Included metastasizing pT1 CRCs (pN+) were matched 1 to 1 , with primary pT1 CRCs with no metastases (pNO) according to the following criteria: Tumor site (right or left colon/rectum), age (maximum tolerance for said match: age +/- 10 years), time of diagnosis (maximum tolerance for said match month of surgery $+/-12$ months) and where possible tumors were also matched by gender. Since inconsistency between clinical reported site and pathological reported site existed for high rectal and low sigmoid CRCs we allowed that low sigmoid colon cancers were matched with high rectal cancers. All matches were reevaluated and criteria for exclusion were the same as mentioned above. Flow chart of inclusion/exclusion is shown in figure 1.

\subsection{Microscopic assessment of high risk features}

All tumors were reassessed by two pathologists using hematoxylin and eosin (HE) stained tumor slides to select the most representative section for immunohistochemical (IHC) staining for high risk features and to select an invasive front area with a tumor cellularity of at least 50\% for miRNA expression analysis. The following high risk features: tumor budding, submucosal lymphovascular invasion (SLI) and submucosal venous invasion (SVI) were evaluated by use of IHC.

SLI and SVI was assessed by IHC staining of lymphatic vessels endothelium with D2-40 and smooth muscle in vessel walls with $\mathrm{H}$-Caldesmon, respectively, and considered positive when single tumor cells or clusters of tumor cells were surrounded by stain positive cells. Tumor budding was assessed by staining of epithelial cells with pan-cytokeratin AE1/AE3, and defined as single tumor cells or clusters of up to 4 nucleus containing tumor cells [5]. Budding was scored in the invasive front by the 10 high-power-field method (HPF, $x 40$ magnification, $0.238 \mathrm{~mm}^{2}$ ) and a cut-off for high grade budding was defined as an average of $\geq 10$ buds/HPF [26]. Where limited tumor areas were available, only the hotspot count (1 HPF, x40, $\left.0.238 \mathrm{~mm}^{2}\right)$ was registered ( 3 out of 56 pT1 tumors). 


\subsection{Immunohistochemistry}

IHC stains were performed at the Department of Pathology at Herlev- and Gentofte Hospital, Denmark, on a DAKO Autostainer Link48 (DAKO, Denmark). Approximately $3 \mu \mathrm{m}$ tumor sections were cut and stained according to manufacturer instructions with pan-cytokeratin AE1/AE3 (DAKO Denmark, Monoclonal Mouse Anti-Human Cytokeratin, Clone AE1/AE3), D2-40 (DAKO Denmark, Monoclonal Mouse Anti-Human Podoplanin, Clone D2-40) and H-Caldesmon (DAKO Denmark, Monoclonal Mouse Anti-Human Caldesmon, Clone h-CD). Immunostains were performed with 4 positive/negative tissue controls. The immunostains are used in the laboratory routine diagnostics and follow the guidelines of Nordic immunohistochemical Quality Control (NordiQC)[27].

\subsection{Tissue sampling and RNA extraction}

From formalin-fixed paraffin-embedded (FFPE) tissue blocks corresponding to the tumor section selected for miRNA analyses, invasive front samples were isolated by use of a 1-2 mm hollow needle. The top $1 / 2-1$ $\mathrm{mm}$ was separated for RNA extraction. Additionally FFPE samples from normal colorectal mucosa and normal stromal tissue from three random CRC patients were sampled.

Total RNA was extracted using the FFPE miRNeasy Kit (Qiagen, Germany) according to manufacturer's instructions and carried out in batches including both LNM positive and negative tumors. Paraffin was removed using xylene (VWR international, France). Tissue samples were digested using $10 \mu$ l proteinase K + $150 \mu \mathrm{l}$ proteinase $\mathrm{K}$ digestion buffer (Qiagen) and lysates were treated with $16 \mu \mathrm{l}$ DNase Booster Buffer and $10 \mu \mathrm{l}$ DNase I stock solution (Qiagen). Total RNA was bound to the RNeasy MinElute spin column membrane and eluted in $20 \mu \mathrm{l}$ RNase-free water resulting in $18 \mu \mathrm{l}$ eluate. Total RNA concentrations were measured using a spectrophotometer (Nanodrop 2000, Thermo Scientific, USA) and eluates were immediately stored at $-80^{\circ} \mathrm{C}$ until microarray analyses.

\section{5 miRNA array analyses}

miRNA array analyses were performed by Medical Prognosis Institute A/S, Denmark (MPI) using Affymetrix GeneChip miRNA Array, according to manufacturer's instructions. In short, 400 ng of miRNA was labeled using FlashTag HSR ${ }^{\mathrm{TM}}$ Biotin RNA Labeling Kit (Genisphere, PA) and hybridized 17 hours at $48^{\circ} \mathrm{C}$ to GeneChip ${ }^{\circledR}$ miRNA 2.0 arrays (Affymetrix, CA). The arrays were then washed and stained with Affymetrix Fluidics Station 450 and scanned on an Affymetrix G7 scanner.

\subsection{Data processing and statistical analyses}

Statistical analyses followed a statistical analysis plan, finalized before the study initiation. Initial data analysis was done using the justRMA function in the Bioconductor package (open source software for bioinformatics), based on R (R Development Core Team, Vienna, Austria, http://www.R-project.org). Intensity measures were adjusted for background and normalized by the quantile method with the resulting expression levels on a log scale base 2 .

The complete data have been deposited in NCBI's Gene Expression Omnibus and are accessible through GEO Series accession number GSE110402[28].

Verification of the arraydata was made by comparison to published datasets on miRNA expression in independent CRC cohorts and by comparison of miR-92a arraydata to qRT-PCR data performed in doublets on a subset of 30 invasive front samples (15 pT1N0 and 15 pT1 N+ CRCs). 
Global miRNA intensity signals were screened using paired t-tests between pT1pN+ and pT1pNO CRCs and other predefined clinico-pathological groups, and taking account of the matched study design. Threshold for significance was set to $\mathrm{p}=0.01$ and subsequent correction for multiple testing was performed using Bonferroni correction.

Analyses of the miR-17/92 cluster were based on expression intensities adjusted for tumor cellularity and tests of hypotheses were performed using general linear models (GLM) adjusting for clinical covariates and taking the pairing into account where relevant. Statistical significance was defined by a $p$-value $\leq 0.05$. The statistical analyses were performed using SAS statistical software packaged (version 9.4, Cary N.C. USA), R (v 3.1.0 R Development Core team, Vienna, Austria, http://www.R-project.org), and IBM SPSS statistical software version 19.

\subsection{Ethics}

The Regional Committee on Health Research Ethics for the Capital Region of Denmark approved the study (Jr.no: H-3-2014-027). The study was approved by and followed the guidelines of The Danish Data Protection Agency (HEH-2014-014), and the Danish Registry of Human Tissue Utilization was consulted before any tissue samples were used for investigation.

\section{RESULTS}

\subsection{Baseline characteristics}

Matching for age was achieved with a median difference of 3 years (range 0-10 years) and matching for time of diagnosis was achieved with a median difference of 8 months between matched patients (range 012 months). Matching for gender was achieved in 25 out of 28 pairs. No significant differences in high risk clinico-pathological features between LNM positive and LNM negative CRCs was observed although a trend was observed for SLI ( $p=0.099)$ and tumor budding $(p=0.091)$ (table 1$)$.

\section{2 miRNA data}

\subsubsection{Global miRNA analyses}

Verification of the expression data was performed by comparing expression intensities with results of other published CRC array expression datasets. Our data showed comparable expression patterns in normal colorectal mucosa as well as comparable fold changes between normal mucosa and CRC tissue (suppl. table 1 and suppl. figure 1). Further, for verification of the array data we compared expression levels of miR-92a analyzed by qRT-PCR, in doublets, in a subset of 30 samples from the invasive tumor front (15 NO CRCs and $15 \mathrm{~N}+\mathrm{CRCs}$ ) and although not significant in this subset of samples, we detected a fold change between $\mathrm{N}+$ CRCs and NO CRCs of 1.23 (95\% Cl: 0.81-1.86, p=0.31), comparable to the 1.28 fold changes detected by miRNA microarray, as described in section 3.2.2

Initial analysis of global miRNA expression with the threshold for significance set to $p=0.01$ showed differential expression of 13 miRNAs in the invasive tumor front of pT1 CRCs with regional LNM when compared to those without (suppl. table 2), however no significant alterations was detected when adjusting for multiple testing. Likewise, we found no significant association between miRNA expression and the presence of other high risk features (SLI, SVI and tumor budding). 


\subsubsection{Analyses of the miR-17/92-cluster}

Following the global analysis, we proceeded to investigate the miR-17/92 cluster. miR-92a, miR-17-5p and miR-20a were among the 30 highest expressed miRNAs in the global array analysis. Of the miR-17/92 members miR-19a had the lowest expression (table 2). All miR-17/92 members except miR-19a showed higher mean expression in CRC tissue compared to normal mucosa, but significant up-regulation were only demonstrated for miR-92a, miR-17-5p and miR-18a (table 2). Expression data showed no significant differences between mean expression levels of the miR-17/92 cluster members in CRC from the right colon, left colon or rectum; neither did we find expression differences between sites when analyzing pN+ and pNO samples separately.

In the tumor tissue, miR-17-3p and miR-92a showed significantly higher expression in the invasive front of tumors with regional LNM compared to tumors without LNM, corresponding to a 1.53 fold higher expression of miR-17-3p (95\% Cl: 1.04-2.24, $\mathrm{p}=0.030)$ and a 1.28 fold higher expression of miR-92a $(95 \% \mathrm{Cl}$ : 1.01-1.68, $p=0.042$ ) in the invasive borders of metastatic tumors. Additionally, we observed a trend for miR$17-5 p$ corresponding to a 1.43 higher expression $(95 \% \mathrm{Cl}: 0.96-2.13, p=0.079)$ in samples from the invasive front of early metastatic CRCs (table 3 ).

Interestingly we found a significant inverse association between expression of miR-19a and tumor budding, corresponding to a 1.55 fold lower expression of miR-19a in tumors classified with high grade tumor budding (95\% $\mathrm{Cl}: 1.13-2.12, \mathrm{p}=0.008)$. Further, we observed a trend for miR-17-3p corresponding to 1.68 times lower expression in tumors with high grade tumor budding ( $95 \% \mathrm{Cl}: 0.98-2.88, \mathrm{p}=0.058)$. No significant associations between miR-17/92 and SLI or SVI were found.

\section{DISCUSSION}

Accurate prediction of regional LNM in endoscopically resected pT1 CRCs is crucial for relevant treatment stratification and herein the identification of patients who will benefit from subsequent surgery.

In the present study we provide evidence for an association between increased tissue expression levels of miR-92a and miR-17-3p and regional LNM in pT1 CRC (table 3), supporting a role for the miRNAs in early metastatic progression of CRC. We have specifically investigated differential expression levels of the miR$17 / 92$ cluster in the invasive tumor front of CRC, an area of particular interest when investigating cancer metastasis. Our results support results of previous studies linking miR-17/92 expression to the EMT-process through repression of the PTEN- mediated PI3K/AKT pathway and thereby initiations of EMT like changes of the epithelial tumor cells $[15,16,29]$.

Despite the high relevance of investigating potential pro-metastatic features in the invasive front of the tumor, such investigations are also challenged by the interplay and varying compositions of neoplastic and non-neoplastic cells in the invasive front micro environment. Manual microdissection of specific invasive front areas enabled estimation of miRNA expression levels of epithelial tumor cells and its local micro environment as a whole, and to minimize bias from varying tumor cell proportion, we adjusted expression intensities for the sample tumor cellularity. However, some impact from miRNA deregulations in the tumor associated stroma cannot be excluded. Nishida et al. demonstrated significant up-regulation of miR-17/92 cluster members in CRC associated stroma and, in line with our findings, showed an association between expression levels of miR-92a in tumor stroma and the presence of distant metastases. However, an 
association to regional LNM was not demonstrated [24]. By semi-quantitative evaluation of miR-92a using miRNA in situ hybridization Tsuchida et al. found significant correlations between high tumor cell miR-92a expression and presence of LNM in colon cancer [21]. Likewise Ke et al. and Zhou et al. demonstrated associations between miR-92a and LNM in CRC tissue from primarily more advanced pT-category tumors $[16,23]$.

From a clinical perspective, assessment of risk of regional LNM, based on miRNA analyses of invasive front tumor tissue, might be challenged by the invasive front not always being easily assessable in lesions that are incompletely endoscopically resected or resected by piecemeal polypectomy. Ke et al. and Zhou et al. were able to demonstrate an association between regional LNM and miR-92a expression in CRC tissue, without specific sampling of the invasive front $[16,23]$, thus indicating that pro-metastatic effects of miR92a is detectable in CRC tumor tissue even without specific sampling. However, such patients with uncertainty about the radicality of the endoscopic resection will probably be offered surgical hemicolectomy including regional lymph nodes subsequent to the endoscopic resection, regardless of the results of risk-assessment for regional LNM.

Like for miR-92a, we found miR-17-3p levels significantly increased in samples from early metastatic tumors. The corresponding strand (miR-17-5p) was similarly increased but the association was not significant at the 0.05 level. In concordance with our findings Ma et al. showed shorter overall survival for patients whose tumors had high expression of miR-17-5p and increased migratory and invasive properties of CRC cells overexpressing miR-17-5p through activation of the $W n t / \beta$-catenin pathway [30]. However, in cell and mouse studies Jiang et al. found that the effect of overexpression of the miR-17/92 cluster on EMT was dependent on the degree of overexpression, since medium levels promoted tumor metastasis through repression of PTEN and activation of the PI3K/AKT pathway, whereas higher levels resulted in inhibition of tumor growth and metastasis through inhibition of the Wnt/ $\beta$-cathenin pathway [31]. Thus, such potential concentration dependent competing cellular effects are a challenge for defining clinical risk estimates based on concentration differences. Interestingly, despite associations between miR-17/92 members and the presence of LNM we also observed a reciprocal association of miR-19a and tumor budding. Previous studies show that miR-19a acts as an oncomir $[32,33]$ and the oncogenic function has been linked to its promotion of tumor angiogenesis [18] and anti-apoptotic/growth-promoting functions [32]. Additionally, suggestions for a role of miR-19a in EMT, migration and metastatic progression of CRC have been proposed [17]. Our findings support a role of miR-19a involvement in the EMT process, but interestingly demonstrate an inverse association with decreased expression in CRCs classified with high grade tumor budding, the morphological phenotype of cells undergoing EMT. However, measured expression intensities of miR-19a were low and thus the observed expression fold changes constitute a small difference in actual expression. In a study of pro angiogenesis effects of the miR-17/92 cluster, Dews et al demonstrated that miR-17/92 mediated inhibition of the TGF $\beta$ pathway caused stimulation of angiogenesis and tumor cell growth [20]. Since TGF $\beta$ plays a dominant role in activating EMT [7], the attenuated TGF $\beta$ signaling could, at least in part, explain the reciprocal association between miR-19a and tumor budding observed in this study.

Our results indicating apparently competing actions of miR-17/92 members in the metastatic process might be a cause of potential concentration-dependent competing actions as demonstrated by Jiang et al [31] or a reflection of interactions on various different targets and thus various oncogenic roles for the miR-17/92cluster members [22]. 
With the intention to identify other miRNAs with predictive value for LNM in PT1 CRC we analyzed associations between presence of LNM and the global miRNA data. With the threshold for significance set at $p<0.01$ we identified 13 miRNAs that were differentially expressed in the invasive front samples of metastatic and non-metastatic tumors (suppl. table 2). However, the fold changes were small and associations were insignificant when adjusting for multiple testing. Previous studies of miRNA heterogeneity demonstrate expression patterns displaying substantial inter-patient and intra-tumor variability including possible functional expression gradients within the tumor $[25,34,35]$. Thus, in a clinical context, the use of miRNA expression levels for differentiating between diagnostic groups and guidance of clinicians in treatment decisions requires extensive demands to sensitivity, specificity and predictive values in order to unambiguously identify the correct treatment for a given patient. Therefore, there is a requirement that miRNA expression differences between groups would be clearly higher than the intratumor and inter-patient variability. In a recent study Kwon Jung et al. identified 66 miRNAs associated to LNM by microarray analyses of 16 pT1 CRCs. Subsequent analyses of 13 selected miRNAs by qRT-PCR in the same cohort and in an independent cohort of 20 patients showed that the selected candidate miRNAs displaying significant differences in one cohort were not significant when investigated in another cohort[14], thus revealing that the inherent heterogeneity in- and between patients, together with biological/technical variation, can make detection of relevant differences difficult. In our study cohort of 56 PT1 CRCs, only three miRNAs initially identified with differential expression between LNM positive and LNM negative CRCs were overlapping with the 66 miRNAs initially identified by Kwon Jung et al. This discordance might be explained by differences in study design (e.g. different array platforms, sampling strategies and the size of the cohort) but might also be explained by one of the major challenges for miRNA studies as mentioned above: Heterogeneous miRNA expression patterns and differences between tissue compartments and patients.

\section{Conclusion}

Accurate prediction of regional LNM in endoscopically resected pT1 CRCs is crucial for treatment stratifications and miRNA profiling of CRC tissue might play a future role in the clinical decision making. We provide evidence for an association between presence of regional LNM and increased expression of miR17-3p and miR-92a, in the invasive front of CRC. Additionally, we find an inverse correlation between miR19a and high grade tumor budding. Taken together our results support a role for the miR-17/92 cluster in early metastatic progression of CRC and calls for further investigation in other independent cohorts.

\section{DisClOSURE/CONFLICTS OF INTEREST}

AH and ITH are employees at Medical Prognosis Institute Aps (MPI). MPI did not offer any financial support or incentives and was not engaged in the scientific content of this paper other than performing the microarray analyses and assisting in the processing of raw expression data. AH and ITH did not have any influence on statistical analyses, interpretation of results or the decision to submit the manuscript for publication. All other authors declare no conflicts of interest. 


\section{ACKNOWLEDGEMENTS}

We thank the laboratory staff at the Department of Pathology and Molecular Unit, Herlev and Gentofte Hospital, in particular Dorte Skriver Jensen and Christina Grønhøj for preparations and immunohistochemically staining of the FFPE tissue slides.

The work was supported by Beckett Foundation (38069/38079), The A.P. Møller Foundation for the Advancement of Medical Science (14-187), Krista and Viggo Petersens Foundation (5793/44), Fabrikant Einer Willumsen Foundation (6000073) and Vald Foersom and Thyra Foersoms Foundation (41452-001). Funding sources had no influence in any part of the study.

\section{Data STATEMENT PAGE}

The complete data have been deposited in NCBI's Gene Expression Omnibus (Jepsen et al., 2018) and upon publication of the manuscript the data are accessible through GEO Series accession number GSE110402 (https://www.ncbi.nlm.nih.gov/geo/query/acc.cgi?acc=GSE110402).

\section{REFERENCES}

1. Ferlay J, Soerjomataram I, Ervik M, et al. GLOBOCAN 2012 v1.0, Cancer Incidence and Mortality Worldwide: IARC CancerBase No. 11 [Internet].Lyon, France: International Agency for Research on Cancer; 2013. Available from: http://globocan.iarc.fr, accessed on 29/06/2016.

2. Siegel R, Desantis C, Jemal A. Colorectal cancer statistics, 2014. CA Cancer.J.Clin. 2014; 64:104-17.

3. Gill MD, Bramble MG, Rees CJ, Lee TJ, Bradburn DM, Mills SJ. Comparison of screen-detected and interval colorectal cancers in the Bowel Cancer Screening Programme. Br.J.Cancer 2012; 107:417-21.

4. European Colorectal Cancer Screening Guidelines Working Group, von Karsa L, Patnick J, Segnan N, et al. European guidelines for quality assurance in colorectal cancer screening and diagnosis: overview and introduction to the full supplement publication. Endoscopy 2013; 45:51-9.

5. Lugli A, Kirsch R, Ajioka Y, et al. Recommendations for reporting tumor budding in colorectal cancer based on the International Tumor Budding Consensus Conference (ITBCC) 2016. Mod.Pathol. 2017; 30:1299-1311.

6. De Smedt L, Palmans S, Andel D, et al. Expression profiling of budding cells in colorectal cancer reveals an EMT-like phenotype and molecular subtype switching. Br.J.Cancer 2017; 116:58-65.

7. Kalluri R and Weinberg RA. The basics of epithelial-mesenchymal transition. J.Clin.Invest. 2009; 119:1420-8. 
8. Suzuki T, Sadahiro S, Mukoyama S, et al. Risk of lymph node and distant metastases in patients with early invasive colorectal cancer classified as Haggitt's level 4 invasion: image analysis of submucosal layer invasion. Dis.Colon Rectum 2003; 46:203-8.

9. Toh EW, Brown P, Morris E, Botterill I, Quirke P. Area of submucosal invasion and width of invasion predicts lymph node metastasis in pT1 colorectal cancers. Dis.Colon Rectum 2015; 58:393-400.

10. Alves A, Panis Y, Mathieu P, Mantion G, Kwiatkowski F, Slim K, Association Francaise de Chirurgie. Postoperative mortality and morbidity in French patients undergoing colorectal surgery: results of a prospective multicenter study. Arch.Surg. 2005; 140:278,83.

11. de Krijger I, Mekenkamp LJ, Punt CJ, Nagtegaal ID. MicroRNAs in colorectal cancer metastasis. J.Pathol. 2011; 224:438-47.

12. Nicoloso MS, Spizzo R, Shimizu M, Rossi S, Calin GA. MicroRNAs--the micro steering wheel of tumour metastases. Nat.Rev.Cancer. 2009; 9:293-302.

13. Schetter AJ, Leung SY, Sohn JJ, et al. MicroRNA expression profiles associated with prognosis and therapeutic outcome in colon adenocarcinoma. JAMA 2008; 299:425-36.

14. Kwon Jung C, Jung SH, Yim SH, et al. Predictive microRNAs for lymph node metastasis in endoscopically resectable submucosal colorectal cancer. Oncotarget 2016; 7:32902-32915.

15. Zhang G, Zhou H, Xiao H, Liu Z, Tian H, Zhou T. MicroRNA-92a functions as an oncogene in colorectal cancer by targeting PTEN. Dig.Dis.Sci. 2014; 59:98-107.

16. Ke TW, Wei PL, Yeh KT, Chen WT, Cheng YW. MiR-92a Promotes Cell Metastasis of Colorectal Cancer Through PTEN-Mediated PI3K/AKT Pathway. Ann.Surg.Oncol. 2015; 22:2649-2655.

17. Huang L, Wang X, Wen C, et al. Hsa-miR-19a is associated with lymph metastasis and mediates the TNFalpha induced epithelial-to-mesenchymal transition in colorectal cancer. Sci.Rep. 2015; 5:13350.

18. Dews $M$, Homayouni A, Yu D, et al. Augmentation of tumor angiogenesis by a Myc-activated microRNA cluster. Nat.Genet. 2006; 38:1060-5.

19. Knudsen KN, Nielsen BS, Lindebjerg J, Hansen TF, Holst R, Sorensen FB. microRNA-17 Is the Most UpRegulated Member of the miR-17-92 Cluster during Early Colon Cancer Evolution. PLoS One 2015; 10:e0140503.

20. Dews M, Fox JL, Hultine $S$, et al. The myc-miR-17 92 axis blunts TGF $\{$ beta\} signaling and production of multiple TGF\{beta\}-dependent antiangiogenic factors. Cancer Res. 2010; 70:8233-46.

21. Tsuchida A, Ohno S, Wu W, et al. miR-92 is a key oncogenic component of the miR-17-92 cluster in colon cancer. Cancer.Sci. 2011; 102:2264-71.

22. Mogilyansky E and Rigoutsos I. The miR-17/92 cluster: a comprehensive update on its genomics, genetics, functions and increasingly important and numerous roles in health and disease. Cell Death Differ. 2013; 20:1603-14. 
23. Zhou T, Zhang G, Liu Z, Xia S, Tian H. Overexpression of miR-92a correlates with tumor metastasis and poor prognosis in patients with colorectal cancer. Int.J.Colorectal Dis. 2013; 28:19-24.

24. Nishida N, Nagahara M, Sato T, et al. Microarray analysis of colorectal cancer stromal tissue reveals upregulation of two oncogenic miRNA clusters. Clin.Cancer Res. 2012; 18:3054-70.

25. Jepsen RK, Novotny GW, Klarskov LL, Christensen IJ, Hogdall E, Riis LB. Investigating intra-tumor heterogeneity and expression gradients of miR-21, miR-92a and miR-200c and their potential of predicting lymph node metastases in early colorectal cancer. Exp.Mol.Pathol. 2016; 101:187-96.

26. Karamitopoulou E, Zlobec I, Kolzer V, Kondi-Pafiti A, Patsouris ES, Gennatas K, Lugli A. Proposal for a 10high-power-fields scoring method for the assessment of tumor budding in colorectal cancer. Mod.Pathol. 2013; 26:295-301.

27. NordiQC. Nordic immunohistochemical Quality Control 2017.

[dataset] 28. Jepsen RK, Novotny GW, Klarskov LL, et al.. MiRNA profiles in invasive front of pT1 colorectal cancer. Gene Expression Omnibus (Jepsen et al., 2018), GEO Series accession number GSE110402 2018; https://www.ncbi.nlm.nih.gov/geo/query/acc.cgi?acc=GSE110402

29. Fang L, Li H, Wang L, Hu J, Jin T, Wang J, Yang BB. MicroRNA-17-5p promotes chemotherapeutic drug resistance and tumour metastasis of colorectal cancer by repressing PTEN expression. Oncotarget 2014; 5:2974-87.

30. Ma Y, Zhang $P$, Wang $F$, et al. Elevated oncofoetal miR-17-5p expression regulates colorectal cancer progression by repressing its target gene P130. Nat.Commun. 2012; 3:1291.

31. Jiang $\mathrm{H}$, Wang $\mathrm{P}$, Wang $\mathrm{Q}$, et al. Quantitatively controlling expression of miR-17 92 determines colon tumor progression in a mouse tumor model. Am.J.Pathol. 2014; 184:1355-68.

32. Olive V, Bennett MJ, Walker JC, et al. miR-19 is a key oncogenic component of mir-17-92. Genes Dev. 2009; 23:2839-49.

33. Lu W, Xu Z, Zhang M, Zuo Y. MiR-19a promotes epithelial-mesenchymal transition through PI3K/AKT pathway in gastric cancer. Int.J.Clin.Exp.Pathol. 2014; 7:7286-96.

34. Eriksen AH, Andersen RF, Nielsen BS, Sorensen FB, Appelt AL, Jakobsen A, Hansen TF. Intratumoral Heterogeneity of MicroRNA Expression in Rectal Cancer. PLoS One 2016; 11:e0156919.

35. Petrovic N, Ergun S, Isenovic ER. Levels of MicroRNA Heterogeneity in Cancer Biology. Mol.Diagn.Ther. 2017. 


\section{Figure CAPTION}

\section{Figure 1.}

Flow diagram illustrating inclusion and exclusion flow.

$\mathrm{n}=$ number of patients; $\mathrm{pT} 1, \mathrm{pN}+, \mathrm{pNO}$ : Pathological tumor and node classification according to the TNM cancer classification of UICC (Union for International Cancer Control), 5.th edition. 


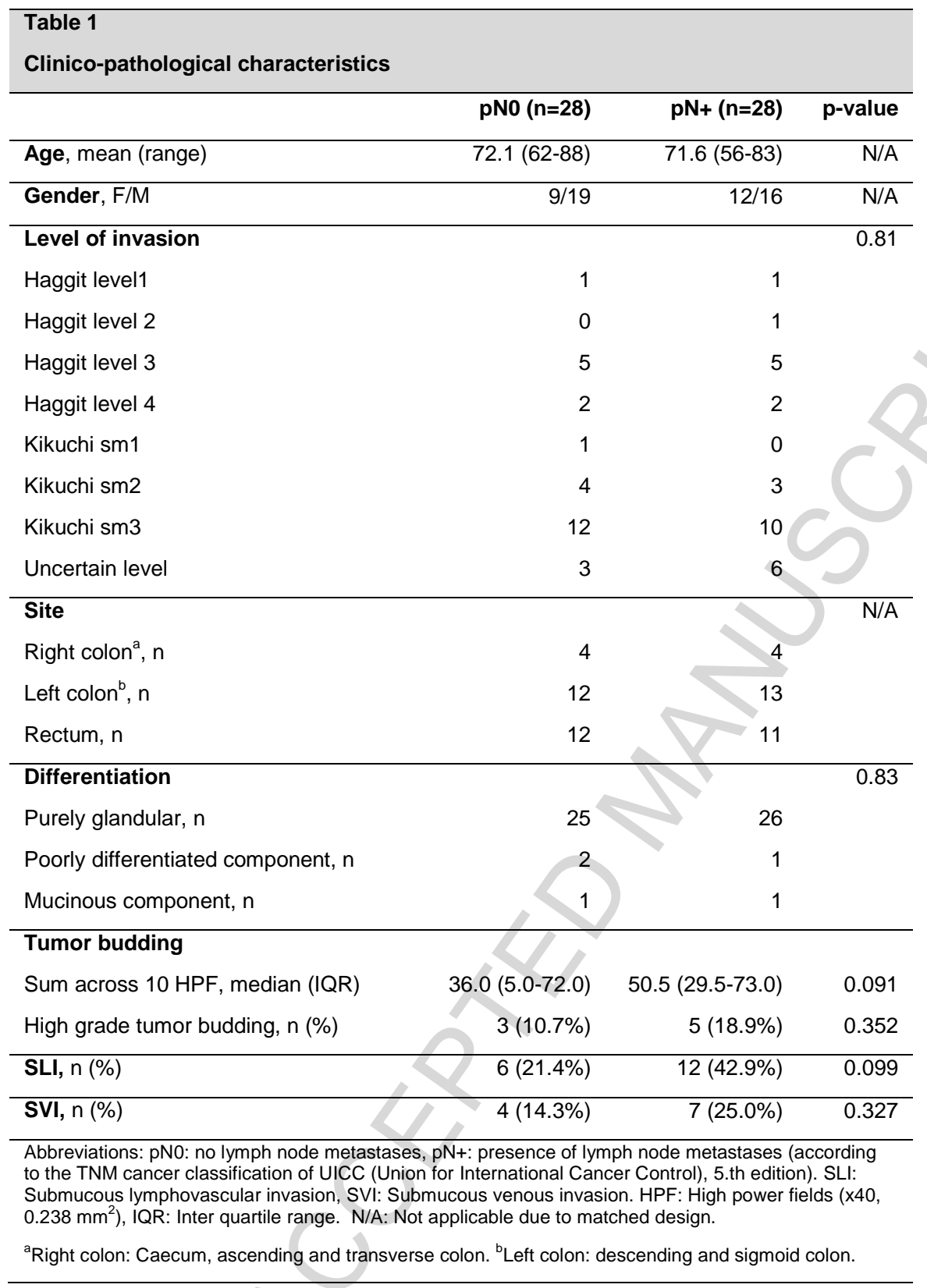




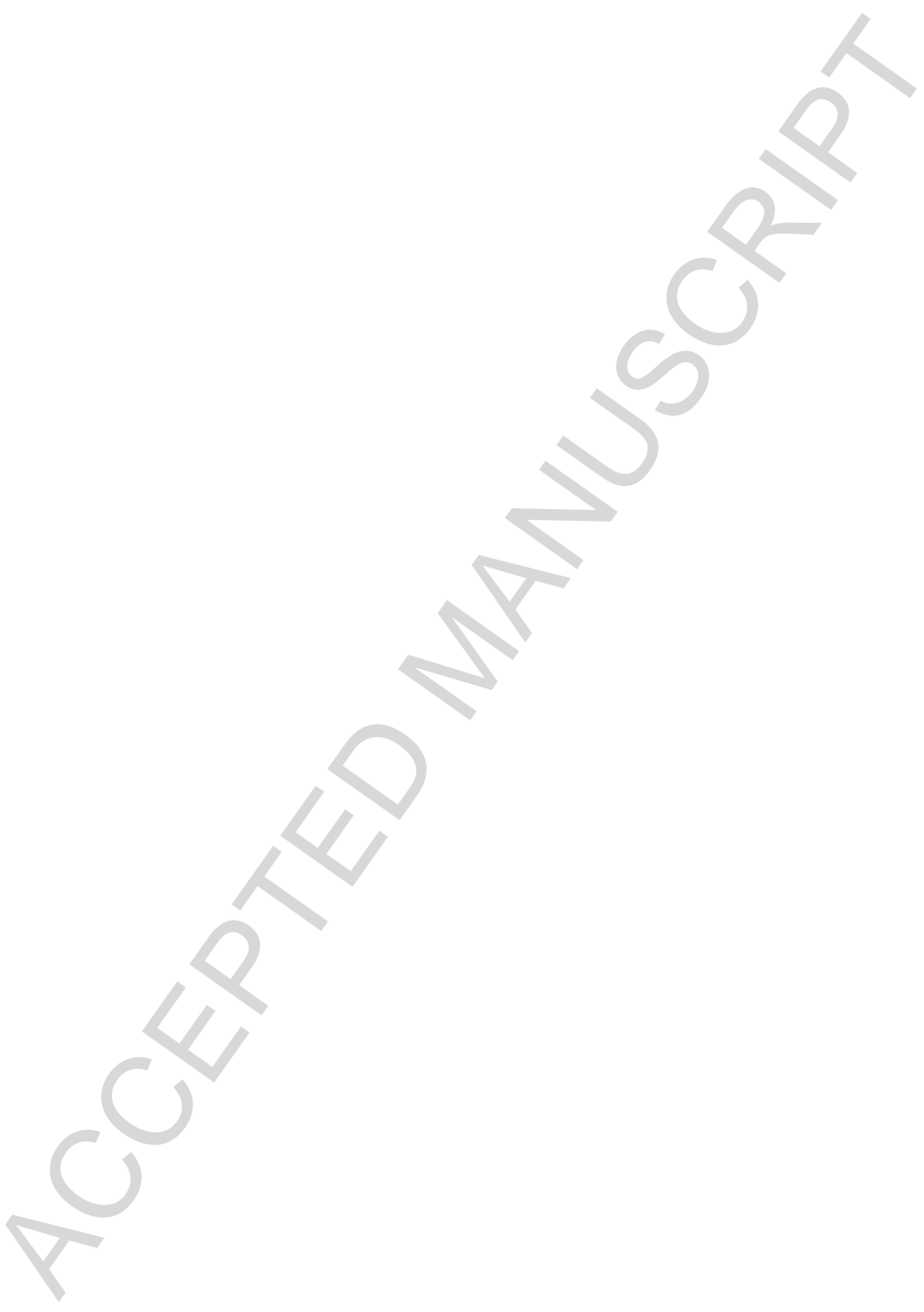




\begin{tabular}{|c|c|c|c|c|c|}
\hline \multicolumn{6}{|c|}{ Mean normalized expression intensities of the $\mathrm{miR}-17 / 92$ cluster } \\
\hline miR-17/92 members & $\begin{array}{l}\text { CRC }^{a} \\
n=56\end{array}$ & $\begin{array}{l}\text { Mucosa } a^{a} \\
n=3\end{array}$ & $\begin{array}{l}\text { Fold } \\
\text { change }^{b}\end{array}$ & $95 \% \mathrm{Cl}$ & p-value \\
\hline hsa-miR-92a & 10.853 & 9.247 & 3.05 & $1.78-5.28$ & $<0.001^{*}$ \\
\hline hsa-miR-17-5p & 9.923 & 8.234 & 3.22 & $1,36-7.53$ & $0.008^{*}$ \\
\hline hsa-miR-20a & 9.173 & 8.035 & 2.19 & $0.82-5.89$ & 0.117 \\
\hline hsa-miR-19b & 6.378 & 5.726 & 1.74 & $0.66-4.61$ & 0.263 \\
\hline hsa-miR-18a & 5.613 & 3.587 & 4.22 & $1.10-16.31$ & $0.037^{*}$ \\
\hline hsa-miR-17-3p & 2.746 & 2.286 & 1.64 & $0.66-4.12$ & 0.285 \\
\hline hsa-miR-19a & 1.674 & 2.159 & 0.76 & $0.44-1.31$ & 0.321 \\
\hline \multicolumn{6}{|c|}{ Abbreviations: CRC: colorectal cancer, hsa-miR: Homo sapiens microRNA. } \\
\hline \multicolumn{6}{|c|}{$\begin{array}{l}{ }^{*} \text { Significant at the } 0.05 \text { level. }{ }^{a} \text { Expression intensities are on Log2 base corresponding to a doubling of } \\
\text { expression intensity per unit increase. }{ }^{b} \text { Expression folds changes between normal mucosa and CRC } \\
\text { tissue. }\end{array}$} \\
\hline
\end{tabular}




\section{Table 3}

Differential expression of miR-17/92 members in LNM + and LNM - pT1 CRCs

\begin{tabular}{llllll}
\hline miR-17/92 members & \multicolumn{2}{c}{$\begin{array}{l}\text { Intensity difference } \\
\text { LNM+ versus LNM- }\end{array}$} & $\begin{array}{l}\text { Fold } \\
\text { change }^{b}\end{array}$ & $95 \% \mathrm{Cl}$ & p-value \\
\hline hsa-miR-92a & upregulation & 0.359 & 1.28 & $1.01-1.68$ & $0.042^{*}$ \\
hsa-miR-17-5p & upregulation & 0.515 & 1.43 & $0.96-2.13$ & 0.079 \\
hsa-miR-20a & upregulation & 0.559 & 1.47 & $0.92-2.37$ & 0.107 \\
hsa-miR-19b & upregulation & 0.390 & 1.31 & $0.81-2.12$ & 0.264 \\
hsa-miR-18a & upregulation & 0.570 & 1.48 & $0.76-2.89$ & 0.239 \\
hsa-miR-17-3p & upregulation & 0.613 & 1.53 & $1.04-2.24$ & $0.030^{*}$ \\
hsa-miR-19a & upregulation & 0.002 & 1.00 & $0.79-1.26$ & 0.989 \\
\hline
\end{tabular}

Abbreviations: CRC: colorectal cancer, hsa-miR: Homo sapiens microRNA, LNM+: lymph node positive, LNM-: Lymph node negative

*Significant at the 0.05 level, ${ }^{a}$ Expression intensities are on Log 2 base corresponding to a doubling of expression intensity per unit increase. ${ }^{b}$ Expression folds changes between LNM+ and LNM- in the invasive tumor front. 


\section{Highlights}

Investigation of miRNA-levels specifically in the invasive tumor front of early CRC

Higher expression of miR-17-3p and miR-92a in the invasive front of early metastatic CRC

Inverse association between miR-19a expression and presence of tumor budding

Results support a role for miR-17/92 in early metastatic progression of CRC 
Resections of pT1 CRC, with regional LNM, consecutively resected and registered in the pathology registry of Herlev- and Gentofte Hospital, Jan 2009 - Jan 2016
pT1pN+
$n=44$

Excluded at review of pathology reports

Age $<50$ years at diagnosis

$n=3$

Synchronous/metachronous CRC $\quad n=3$

Other infiltrating cancers $\quad n=1$

Excluded during histopathological reassessment

Uncertain/advanced tumor stage $\quad n=1$

No/ insufficient tumor tissue available $\quad n=8$

Cohort of early metastasizing CRCs,

pT1pN+

$n=28$

pT1pN+ patients were matched 1 by 1 , with primary early non-metastatic CRCs (pT1pNO) Match criteria: age, year of diagnosis tumor site .and gender.

Final matched cohort,

pT1pN+/pT1pNO $n=56$ 